Microfluidics

\section{DNA microarrays}

\section{get the shakes}

Anal. Chem. 75, 1911-1917 (2003)

At the microscopic scale, water acts like a highly viscous liquid and mixing is slow. This is a problem for microfluidic chemistry, as it slows down the rate of encounters between reagents. Genetic analysis of DNA on microchip arrays, which relies on target and probe strands of DNA getting together, can take hours because this hybridization process is limited by the rate of strand diffusion.

Robin Hui Liu and colleagues have shown that hybridization can be speeded up by using the acoustic vibration of tiny air bubbles to stir the liquid. They position a plastic film just above a glass microarray chip, perforated with tiny holes that each contains an air bubble. A piezoelectric oscillator makes the bubbles vibrate, creating a 'microstreaming' circulation of the liquid between the plastic film and the glass slide. This speeds up hybridization roughly fivefold, so that the microarray read-out signal is correspondingly stronger after two hours. And the resulting signal is much more uniform over the chip. This simple, cheap technique could be a boon to high-throughput microarray-based genetic screening. Philip Ball

\section{Sensory transduction}

\section{Feel the heat}

Science 300, 1284-1288 (2003)

Bite into a chilli and you'll soon know about it. The burning sensation that follows is caused by capsaicin, a compound that stimulates receptors found on the surface of sensory nerve cells in the tongue and elsewhere. These so-called TRPV1 receptors form part of a warning system, signalling the presence of various potentially harmful stimuli, including low $\mathrm{pH}$ and noxious heat. Elizabeth D. Prescott and David Julius now show how the sensitivity of TRPV1 receptors is regulated to ensure that we detect and respond to such threats appropriately.

The researchers looked at the interaction of the TRPV1 protein with the lipid molecule $\mathrm{PIP}_{2}$, which can hold the receptor in an inhibited state. By modifying the structure of TRPV1 in various ways, the authors identified the site at which PIP $_{2}$ binds. Structural changes that weakened the interaction of TRPV1 with $\mathrm{PIP}_{2}$ increased the response of the receptor to chemical and thermal triggers, reducing the threshold for detecting these stimuli.

Prescott and Julius suggest that the strength of the TRPV1-PIP ${ }_{2}$ interaction sets the threshold for detecting the onset of

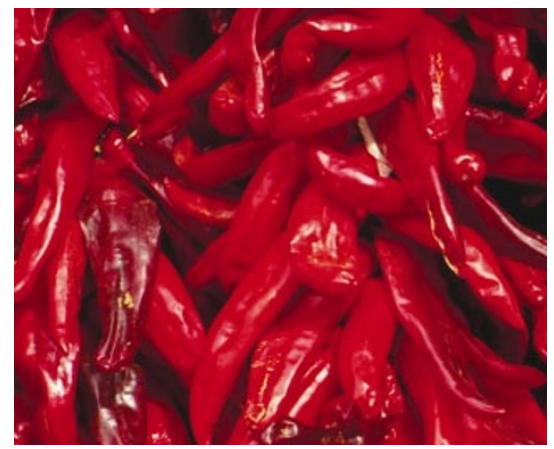

tissue damage. They also propose that the $\mathrm{PIP}_{2}$-binding site represents a target for treatments aimed at altering the sensitivity of nerve cells to damaging stimuli in normal and pathological conditions. Rebecca Craven

\section{Earth science}

\section{Core effect}

Geology 31, 415-418 (2003)

Life on Earth 2.45 billion years ago was harsh, but it was safely swaddled in the same protective magnetic field that we enjoy today, according to A. V. Smirnov and colleagues.

The magnetic field is generated by the dynamo effect of molten iron sloshing around in the Earth's core; by repelling charged particles, the magnetic field shields the planet from harsh radiation from space. But it's difficult to know when the Earth's dynamo turned on. The best method for finding out is to measure the magnetic field remaining in rocks to infer the strength of the magnetic field that ran through them in days past. But this picture is often clouded by the weathering and mixing of rocks over time.

Smirnov et al. have measured the magnetic field in 2.45-billion-year-old rocks from Karelia in Russia. To overcome the problem of weathering, they looked at individual crystals of rock - the first time this has been done with rocks of this vintage. They find that the magnetic field present 2.45 billion years ago was similar to that of today. This implies that the Earth's solid iron inner core - believed to stabilize the dynamo - had started to form before that time. If correct, this is an important yardstick for Earth researchers. Tom clarke

\section{Cell biology}

\section{Rafting for dear life}

Immunity 18, 655-664 (2003)

Cell membranes are made up of a sea of lipid molecules, in which so-called rafts are afloat — these are membrane patches that are high in sphingolipids and cholesterol. Rafts can serve as launching pads for transmitting signals into the cell. Daniel F. Legler et al. now report that, when activated, a receptor for the tumour-necrosis factor- $\alpha$ (TNF- $\alpha$ ) protein is recruited to rafts, where it launches a molecular signalling cascade necessary for the cell to survive.

Usually, the activation of this receptor (TNF receptor 1) by TNF- $\alpha$ leads to the production of anti-inflammatory and survival factors - but occasionally it results in programmed cell death. Legler and colleagues found that, following activation and adrift on its raft, the receptor attracted various signalling proteins, which formed a complex that could transmit the signal through the cell. The receptor and one of the proteins in the complex also rapidly became modified - most probably with ubiquitin molecules. These molecules label proteins for rapid destruction and may be necessary to quench the signal at some point.

Most importantly, disrupting the organization of the rafts switched TNF- $\alpha$-induced signalling from cell survival to cell death. The role of rafts in TNF- $\alpha$ signalling is not always clear-cut, but here it looks as if the cells have to raft for dear life to survive. Marie-Thérèse Heemels

\section{Cancer}

\section{Genetic prescription}

\section{Clin. Cancer Res. 9, 1611-1615 (2003)}

A patient's genetic make-up may affect how well they respond to anti-cancer treatments. According to Victor Cohen et al., people with colorectal cancer respond better to a common type of chemotherapy if they have a particular amino-acid sequence variation in the enzyme methylenetetrahydrofolate reductase (MTHFR), which is involved in folic acid metabolism.

Colorectal cancer is commonly treated with fluoropyrimidine compounds, which affect the composition of the pool of folic acid derivatives inside cells. This pool is also affected by a sequence variation in MTHFR (where an alanine amino acid is changed to a valine), which reduces the activity of the enzyme. It has been suggested that this variation is associated with a decreased risk of colorectal cancer.

Cohen and colleagues wondered whether the variation also correlates with a better response to fluoropyrimidines. They carried out an initial study of 43 patients with colorectal cancer, 26 of whom carried the valine variant of MTHFR. Tumours were less likely to develop further in patients with this variant than in patients with the alanine variant.

The findings point to 'personalized' treatments, and to a possible new drug target. But first a larger-scale, randomized study is needed to find out how accurately the sequence variation predicts the response to treatment and whether it has other long-term consequences.

Camilla Carlsson 